\title{
Cognitive Fatigue in Repetitive Learning Task
}

\author{
Ping Ren* \\ University of Rochester Medical Center, USA \\ *Corresponding author: Ping Ren, School of Nursing, University of Rochester Medical Center, Rochester, NY, USA
}

Submission: July 11, 2018; Published: August 06, 2018

\section{Editorial}

The ability to learn is fundamentally important to the survival of all animals. To better adapt to the changing environment and daily challenges, people need to learn new skills and knowledge by practicing repetitively. The learning effect mainly depends on brain plasticity during training, and decreases over time due to becoming tired, losing energy, and failing to sustain attention. Cognitive fatigue (CF) is about the decrement in mental effort or self-motivation when engaging in cognitively demanding tasks. Due to inevitable CF during repetitive training, the task performance cannot be improved or maintained permanently. In addition, CF has been found to be a considerable symptom in many psychiatric disorders, such as multiple sclerosis (MS), Alzheimer's disease, and Parkinson disease. Comparing with numerous studies on the mechanism of learning progress, we know much less about fatigue, especially the relationship between learning and CF.

Many human and animal studies have shown the decreased activity of multiple brain regions during repetitive training, which reflects learning effects. While several neuroimaging studies reported that CF is associated with increased activation in several brain areas, including basal ganglia, frontal regions and parietal regions during a speed assessing task in MS patients. Boksem and his colleagues suggest mental effort invested in cognitively demanding tasks plays a critical role in the experience of CF. In their model, the improvement of objective performance from cognitive tasks is often accompanied with the generation of CF. Thus, the consumption of brain resource during a repetitive task may decrease at the beginning (better performance) and increase after an inflection point (unchanged or worse performance) (Figure 1). This simplified model reflects the transition of neural activity during a repetitive task, however, it doesn't mean learning and CF are separate progress in time domain. In fact, the $\mathrm{CF}$ and learning both exist during the entire training progress, showing progressively decreased learning effect and increased CF over time. The decreased brain activity at the beginning may reflect more learning effect and less $\mathrm{CF}$, while the increased brain activity later may reflect less learning effect and more CF (Figure 1).

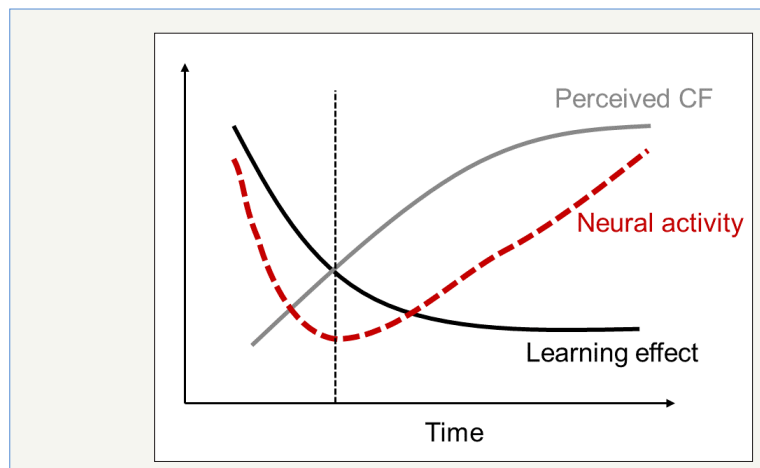

Figure 1: A schematic model of fatigue-learning progress during a repetitive task.

However, the model only involves change of neural activation in 'time domain', which may be not enough. The changes in 'spatial domain' need to be considered as well, that extra brain regions may be recruited to compensate CF-induced performance decline. An electroencephalography (EEG) study has shown that anterior frontal brain activity is recruited to compensate for fatigue-induced impairments in the primary task-related networks. To better understand CF, it is important to find more objective CF biomarker, rather than depend on self-report assessments. Lin and her colleagues have shown the difference of heart rate variability in people with low and high $\mathrm{CF}$, which shed a light on the mechanism of $\mathrm{CF}$. With novel biomarkers of $\mathrm{CF}$, it may help disentangle the CF from learning progress in future research. 
Creative Commons Attribution 4.0 International License

For possible submissions Click Here

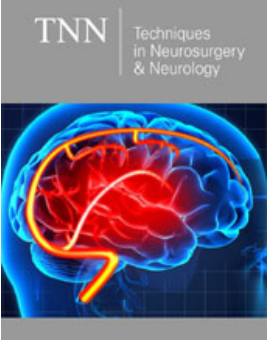

\section{Techniques in Neurosurgery \& Neurology}

Benefits of Publishing with us

- High-level peer review and editorial services

- Freely accessible online immediately upon publication

- Authors retain the copyright to their work

- Licensing it under a Creative Commons license

- Visibility through different online platforms 